\title{
Identification of Two Major Rice Sheath Blight Resistance QTLs, qSB1-1HJX74 and qSB11HJX74, in Field Trials Using Chromosome Segment Substitution Lines
}

\author{
Yajun Zhu, Shimin Zuo, Zongxiang Chen, Xingguang Chen, Gang Li, and Yafang Zhang, The Key Laboratory of Crop Genetics \\ and Physiology of Jiangsu Province, The Key Laboratory of Plant Functional Genomics of the Ministry of Education, College of Agri- \\ culture, Yangzhou University, Yangzhou 225009, China; Guiquan Zhang, The State Key Laboratory for Conservation and Utilization \\ of Subtropical Agro-bioresources, South China Agricultural University, Guangzhou, China; and Xuebiao Pan, The Key Laboratory of \\ Crop Genetics and Physiology of Jiangsu Province, The Key Laboratory of Plant Functional Genomics of the Ministry of Education, \\ College of Agriculture, Yangzhou University, Yangzhou 225009, China
}

\begin{abstract}
Sheath blight $(\mathrm{SB})$ is among the most destructive rice (Oryza sativa) diseases worldwide. SB resistance (SBR) is controlled by quantitative trait loci (QTL). Only a few SB resistance QTLs were confirmed previously in field trials that were independent of morphological traits, a crucial factor in plant breeding. Here, we employed 63 chromosome segment substitution lines (CSSLs) to identify SBR QTLs derived from 'HJX74'. Importantly, these CSSLs all carried the same genetic background as 'HJX74', except in the substituted segment introgressed from susceptible 'Amol3(sona)'. In contrast to most reports that mapped SBR QTLs under complex genetic backgrounds, this approach allowed many CSSLs to consistently retain the agronomic traits of
\end{abstract}

Abstract

Zhu, Y., Zuo, S., Chen, Z., Chen, X., Li, G., Zhang, Y., Zhang, G., and Pan, X. 2014. Identification of two major rice sheath blight resistance QTLs, $q S B 1-1^{H J X 74}$ and $q S B 11^{H J X 74}$, in field trials using chromosome segment substitution lines. Plant Dis. 98:1112-1121.

'HJX74' with moderate resistance, giving the needed high reproducibility in SBR scoring. We have identified five SBR QTLs in field tests. Two of them, $q S B 11^{H J X 74}$ and $q S B 1-1^{H J X 74}$, conferred the greatest reduction in SB ratings by approximately 0.9 to 1.2 on a 0 to 9 scale. $q S B 11^{H J X 74}$ exhibited nearly perfect recessive heredity, whereas $q S B 1$ $1^{H J X 74}$ showed dominant heredity. Using a secondary $\mathrm{F}_{2}$ population and overlapping substitution segment lines, we further mapped $q S B 11^{H J X 74}$ and $q S B 1-1^{H J X 74}$ to regions of approximately 430 and $930 \mathrm{~kb}$, respectively. The results will accelerate the rice breeding process for resistance to SB disease.
Rice (Oryza sativa L.) is one of the most important food crops in the world, feeding more than $50 \%$ of the global human population. As one of the most severe rice diseases, sheath blight (SB) causes substantial losses of rice production annually, especially in intensively managed production systems $(3,25)$. Under the most favorable environmental conditions for disease development, rice SB can cause yield losses up to $50 \%(18,25)$. The causal agent of rice SB is the soilborne, necrotrophic fungal pathogen Rhizoctonia solani J.G. Kuhn 1858, which has a wide host range $(3,44)$. Since the second Green Revolution, with the excessive application of nitrogen fertilizer and the wide geographic utilization of semi-dwarf and high tiller-number rice varieties, SB disease has become increasingly severe. In China, SB affects approximately 15 to 20 million ha of rice and causes a yield loss of approximately 6 million tons per year (2). In some rice-growing regions of southern China, SB has become the most destructive disease and has caused even greater yield losses than rice blast disease $(12,49)$. Developing resistant rice cultivars is the most economical and efficient way to control this disease. However, since rice resistance to SB disease is a typical quantitative trait that readily interacts with a range of environmental factors, progress in conventional breeding for SB resistance has been slow. For instance, nearly all recently released, commercialized rice varieties from most rice production regions in China were found to be susceptible or highly

Corresponding authors: Shimin Zuo, E-mail: smzuo@yzu.edu.cn; and Xuebiao Pan, E-mail: shuidao@yzu.edu.cn

* The $\boldsymbol{e}$-Xtra logo stands for "electronic extra" and indicates that Figure 3 appears in color online.

Accepted for publication 18 February 2014.

http://dx.doi.org/10.1094/PDIS-10-13-1095-RE

(c) 2014 The American Phytopathological Society susceptible to rice SB $(24,43,46)$. In general, rice SB disease occurs most severely at the later tillering and heading developmental stages in field-grown rice (51). Therefore, developing rice varieties with high levels of resistance expressed at these phenological stages is of crucial importance to manage SB effectively.

To date, approximately 50 SB-resistant quantitative trait loci (QTLs; hereafter referred to as SBR QTLs) have been reported $(7,9,14,16,36,48)$. However, only a few of them have been confirmed to have clear SBR effects at the adult stage in the field and which have clearly been shown to be not associated with morphological traits by using near-isogenic lines (NILs) $(16,48)$. Most of the previous studies used RILs (recombinant inbred lines), $F_{2}$ clonal lines, or DH (double haploid) populations to identify SBR QTLs (48). Since in these populations the morphological traits and genetic backgrounds vary widely among lines, the resistance effects of SBR QTLs detected in these studies could not be accurately evaluated $(15,36)$. In addition, whether these SBR QTLs are associated with morphological traits remains largely unknown. Therefore, although using these populations with complex genetic backgrounds repeatedly identified some of these SBR QTLs, they still need to be further confirmed by using NILs. Increasingly more studies are evaluating the SB resistance of rice at the seedling stage in greenhouses in order to minimize the effects of morphological differences on resistance that are associated with the different genetic backgrounds $(15,37)$. Although these improvements are considered to be helpful for evaluating the SBR phenotype of an SBR QTL, breeders desire SBR QTLs with clear resistance at the adult stage in field $(26,36,48)$. Also, inconsistent results have been reported between SBR QTLs identified at the seedling and the adult stages (47). This means that not all SBR QTLs identified at the seedling stage in a greenhouse will perform well at the adult stage in the field. Therefore, there remains a lack of SBR QTLs that are confirmed to confer clear resistance effects at the adult stage in the field and which are independent of morphological traits, although the marker-assisted selection (MAS) approach for SBR QTLs has been considered a feasible strategy to develop SB-resistant variety (6). 
The strategy of using chromosome segment substitution lines (CSSLs), which were constructed by crossing and advanced crossing based on MAS, has been widely used in recent studies to identify and characterize QTLs $(11,17)$. All CSSLs in a population share an almost identical genetic background inherited from their recurrent parent. Each CSSL differs only at the single or a very few chromosomal segments derived from the donor (11,23). Each CSSL can also be considered as a NIL to the recurrent parent. As a result, each QTL located in a certain chromosome segment can be considered as a single Mendelian factor because of the minimized genetic background noise (20). After the primary mapping, many QTLs of interest can be readily fine-mapped and characterized by constructing a secondary $\mathrm{F}_{2}$ population using the selected CSSLs or NILs $(26,31,35,38,39)$. In rice SB resistance, to date, only a few SBR QTLs have been characterized by using NILs and introgression lines (ILs, like CSSLs) that include $q S B-7^{T Q}, q S B-9^{T Q}$ (or $q S B$ $\left.9^{J 85}\right), q S B-11^{L E}$, and $q S B-12^{T Q}(33,36,48)$. Among these studies, the $q S B-9^{T Q}$ showed clear resistance at both the seedling stage in the greenhouse and at the adult stage in the field $(36,41)$, and displayed a significantly greater effect than $q S B-12^{T Q}$ in the field $(36,41)$; on the contrary, the $q S B-11^{L E}$ only showed resistance at the adult stage (47). In addition, the $q S B-9^{T Q}$ and $q S B-12^{T Q}$ exhibited completely dominant heredity, whereas $q S B-11^{L E}$ showed incomplete dominant heredity $(36,41,47)$. These findings presented information critical to breeders in order to maximize the benefits of these SBR QTLs in an applied breeding program (49). For instance, $q S B-11^{L E}$ is not appropriate for hybrid rice breeding because its contribution is smaller under heterozygous conditions than under homozygous conditions $(47,48)$. To the best of our knowledge, except for the few SBR QTLs mentioned above, all other SBR QTLs identified so far are still lacking this critical information, which thereby hinders their effective utilization.

Through advanced backcrossing and MAS, Akshay and Zhang (1) developed a set of 63 CSSLs by using the indica variety HuaJingXian74 (HJX74) as the recurrent parent and the indica variety 'Amol3(sona)' as the donor parent. 'HJX74' is an important commercialized variety in southern China with high grain yield potential and good quality and has been widely used as one of the important breeding parents in indica rice breeding. All of these CSSLs had similar genetic backgrounds with 'HJX74', and the substitution chromosome segments in these lines together nearly covered the whole genome of 'Amol3(sona)' (1). Since each of the CSSLs only contained a very few segments from the donor parent, these lines represent an excellent population for QTL mapping and effects estimation (1). In our previous field tests, we found 'HJX74' exhibited moderate resistance to SB in the field, whereas 'Amol3(sona)' was highly susceptible to SB (unpublished data). Therefore, in the present study, we employed this population to identify SBR QTLs with resistance at the adult stage in the field and that showed no linkage to morphological/developmental traits. We also determined the genetic mode of inheritance of the SBR QTLs having greater effects on resistance and narrowed down the regions of the major SBR QTLs for increasing MAS efficiency. Results in this study will directly accelerate the utilization of these SBR QTLs from 'HJX74' in breeding practice, especially in southern China because 'HJX74' is currently a widely used breeding parent and SB disease has become the most serious rice disease in this region.

\section{Materials and Methods}

Plant materials. The rice lines used in this study included the recipient indica parent, 'HJX74' (kindly provided by Guiquan Zhang from South China Agricultural University), the donor parent 'Amol3(sona)', and 63 chromosome segment substitution lines (CSSLs) carrying the genetic background of 'HJX74'. 'HJX74' is a high-yielding indica rice cultivar widely grown in South China (authorized certificate number in China: CNA20050527.0) (http://www.ricedata.cn/variety/) that exhibits moderate resistance to the SB disease. 'Amol3(sona)' is a long-grain indica rice from Iran that is highly susceptible to sheath blight. The combined donor chromosome segments in the set of CSSLs cover almost the entire rice genome of 'Amol3(sona)'. Each CSSL has been verified to carry one single chromosome fragment from the donor parent (1).

Field design. The above materials were planted in the experimental farm at the Agriculture Department of Yangzhou University, China (located 32.24 degrees north latitude and 119.26 degrees east longitude). For all homozygous lines and two $F_{1}$ lines, the experiments were conducted using a randomized complete block design with three replications in each year. The distances between rows and individuals in each row were 35 and $20 \mathrm{~cm}$, respectively.

In 2007, each replication included the population of a set of 63 CSSLs and two parents. Each line was planted in a plot that had three rows, with 12 individuals per row. The 10 center plants in the middle row of each plot were used for SB inoculation. The 10 plants from the first row (noninoculated row) of each line were selected for agronomic trait measurements. In 2008, 52 out of the 63 CSSLs were removed because of their obvious differences from 'HJX74' in morphological traits or the same SB resistance level as 'HJX74'. The remaining 11 CSSLs and two parents were planted in each replication. Each line was planted and inoculated the same way as in 2007 .

In 2009 and 2010, the field inoculation tests included two CSSLs (CSSL-P271 and CSSL-P272); the recurrent parent 'HJX74', and two $F_{1}$ lines produced independently by crossing CSSL-P271 and P272 with 'HJX74', respectively. These two CSSLs were further evaluated in the field because they were confirmed to be associated with two major SBR QTLs derived from 'HJX74'. Therefore, each replication included five lines. Each line was planted in the plot containing five rows with 12 plants per row. The 10 center plants in each of three central rows were used for inoculation. Therefore, the inoculation scale for each line in each replication was increased up to three times compared to 2007 and 2008. In addition, 10 plants from the first row (noninoculated row) of each line were used to record the agronomic traits.

In 2010, the inoculation experiment contained approximately $600 \mathrm{~F}_{2}$ individuals derived from a cross between the recurrent parent 'HJX74' and CSSL-P271 (HJX74 × P271). In this experiment, all $\mathrm{F}_{2}$ plants were transplanted into a plot without replication. The plot consisted of 54 rows with 12 plants per row. 'HJX74' was planted in the first two rows and the last two rows to serve as the border rows. The 10 center plants in each row were used for inoculation. Surrounding the inoculated plot, a border region containing 'HJX74' was set up with five plants per row. The border region and inoculation region were $40 \mathrm{~cm}$ apart to facilitate disease scoring. The distances between plants were the same as above.

In 2012, four lines, 'HJX74', 'Amol3(sona)', CSSL-P272, and CSSL-P274, were repeatedly evaluated in field. The field design and management were the same as in 2009 and 2010 for the five lines.

Fungal inoculation and symptom scoring. $R H-9$, a $R$. solani strain with high virulence, was obtained from the Plant Protection Institute of Jiangsu Academy of Agricultural Science, Nanjing, China. SB inoculation using thin, woody matchsticks at the late tillering stage was performed as described previously $(41,45$, 48,49).

The SB disease scores were recorded at 30 days after heading (DAH) using the scale described by Zuo et al. (51). The disease score of each plant was presented as the average scores of the three most severely diseased tillers on a plant.

Development of molecular markers. Each CSSL line, P271 and P272, contained an introgressed chromosome segment from the susceptible donor parent 'Amol3(sona)'. Based on the molecular markers tagged in each line located on the IRGSP Build5 pseudomolecules of rice variety Nipponbare, we conclude that the introgressed segment in CSSL-P271 covers the interval between markers PSM418-RM224-RM144 on chromosome 11 and is estimated at approximately $3,500 \mathrm{~kb}$ in length. The introgressed segment in CSSL-P272 covers the interval between markers RM151RM490-RM259 on chromosome 1 and is estimated at approximately $1,890 \mathrm{~kb}$ in length. 
In each introgressed region, several new polymorphic markers including public SSR markers and InDel (insertion/deletion) markers were developed. For developing polymorphic SSR markers, we first downloaded and synthesized the public SSR primers in the region of interest from the GRAMENE marker database (http://www.gramene.org), then confirmed their polymorphism between the parents by separating PCR products on $4 \%$ agarose gel. For developing In/Del markers, we first downloaded the DNA sequence of the reference variety Nipponbare in the region of interest from the rice genome database (http://rgp.dna.affrc.go.jp). Then, using BLASTn provided on NCBI (http://blast.ncbi.nlm.nih. gov/Blast.cgi) to find InDel DNA diversity between the downloaded sequence and the sequence from the indica variety 93-11, we designed primers on both sides of the candidate InDel point using the Primer Premier 5.0 software (http://www.tucows.com/ preview/205452). The primers were then used to amplify total DNA of 'HJX74' and 'Amol3(sona)', and the products were separated by $4 \%$ agarose gel to confirm the polymorphism. The confirmed polymorphic primers were used as the new In/Del markers for the subsequent experiments. The details for developing In/Del markers and designing primers were described by Zuo et al. (47) and Ji et al. (13), respectively.

DNA extraction and genotyping. Total genomic DNA was extracted following the method described by Tan et al. (33). PCR reactions were performed as described previously (41).

Investigation of agronomic traits. In this study, seven agronomic traits for each rice line, including heading date (HD), plant height (PH), flag leaf length (FLL), flag leaf width (FLW), tiller angle (TA), tiller number (TN), and panicle length (PL), were measured and recorded. HD was recorded as the number of days between sowing and heading. PL was measured at 40 days after heading on 10 plants per rice line. The other five traits were measured at 10 days after heading on 10 plants from the first row (without inoculation) in each plot.

Data analysis. The phenotypic value of each line, the traits measured in this study including SB disease score and morphological traits, in each replication was represented by the average phenotypic values of 10 (2007 and 2008) or 30 (2009, 2010, 2012) plants. The average phenotypic value of each replication was used in ANOVA (analysis of variance) packaged in SPSS16.0. The rice lines and years were set as two fixed factors. Univariate procedure in general linear model was used to reveal the differences of the tested traits (SB score or agronomic traits) between 'HJX74' and 'Amol3(sona)' and each of 11 CSSLs evaluated in 2007 and 2008. For those CSSLs showing significant $(P \leq 0.05)$ difference on resistance compared to the 'HJX74' control by two-way ANOVA using Dunnett's method, the regions of substitution segments in these CSSLs were concluded to harbor SBR QTLs $(8,40)$. The additive effect and the percentage of phenotypic variation ex- plained of each SBR QTL were estimated according to the method described by Eshed and Zamir (8). Additive effect was half of the difference between the phenotypic value (here referred to as SB disease score) of each homozygous CSSL and 'HJX74'. Percentage of phenotypic variation was calculated as $=$ (additive value/phenotypic value of 'HJX74') $\times 100 \%$. The difference of SB resistance and measured morphological traits among five lines evaluated in 2009 and 2010 ( 2 years data combined), and among four lines in 2007, 2008, and 2012 (3 years data combined), were revealed by two-way ANOVA using Tukey's multiple comparison in a general linear model. The difference of SB disease rating of each independent year among four lines in 2007, 2008, and 2012 were analyzed by one-way ANOVA and means separated using Tukey's approach, respectively.

For QTL mapping, a molecular linkage map for the chromosome substitution region was constructed by using 489 secondary $F_{2}$ individuals using the QGA2.0 software (5). The composite interval mapping (CIM) in WinQTLCart2.5 (34) was used to further map one major SBR QTL. The scan interval was set at $2 \mathrm{cM}$ using the Kosambi mapping function with an automatic selection of peak markers for putative QTL as cofactor. The logarithm of the odds (LOD) score of 3.0 was used in this study for determining a QTL. The estimations of the position, genetic effects, and percentage of phenotypic variation of QTL were performed at the significant LOD peak in the region. For the second SBR QTL, the map-based cloning strategy was used to further narrow down its region $(38,47)$.

\section{Results}

Phenotypes of parents and chromosome single segment substitution lines. The phenotypes of the two parents with regard to resistance to SB disease were evaluated in field trials conducted using a randomized complete block design in 2007 and 2008. The average SB scores for 'HJX74' was significantly lower than that of 'Amo13(sona)' (Table 1). 'HJX74' had an average SBR score of 3.97, which indicated a moderate resistance level in the 0 to 9 rating scale, whereas 'Amo13(sona)' had an average SBR score of 7.31, indicating complete susceptibility.

The resistance of the 63 CSSLs to SB disease was preliminarily evaluated in 2007. The SB disease scores for all CSSLs ranged from $2.65 \pm 0.25$ to $5.44 \pm 0.11$. The scores of controls 'HJX74' and 'Amol3(sona)' were $3.92 \pm 0.19$ and $7.13 \pm 0.12$, respectively (Fig. 1). Compared with 'HJX74', 13 CSSLs displayed various HD and $\mathrm{PH}$ values, whereas the remaining 50 CSSLs exhibited almost the same morphological and developmental traits as 'HJX74' in the field (data not shown). To identify the SBR QTLs that were not associated with morphological and developmental traits, we removed these 13 CSSLs from SB disease data analysis. For the remaining 50 CSSLs, we found that 11 of them showed levels of resistance different from that of 'HJX74' (data not shown) in the

Table 1. Differences between 10 chromosome segment substitution lines (CSSLs) and 'HJX74' on sheath blight resistance (SBR) and morphological and developmental traits revealed by using 2-year data $\left(2007\right.$ and 2008) ${ }^{\mathrm{z}}$

\begin{tabular}{|c|c|c|c|c|c|c|c|c|c|}
\hline Material & $\begin{array}{c}\text { Average SB } \\
\text { score } \pm \text { SD }\end{array}$ & $\begin{array}{l}\text { SBR QTL } \\
\text { replaced }\end{array}$ & $\begin{array}{l}\text { Chromo- } \\
\text { some }\end{array}$ & $\begin{array}{c}\text { Additive } \\
\text { effect }\end{array}$ & $\begin{array}{l}\% \text { Additive } \\
\text { effect }\end{array}$ & PH (cm) & TA $\left(^{\circ}\right)$ & TN & $\begin{array}{c}\text { HD } \\
\text { (day) }\end{array}$ \\
\hline 'HJX74'(control) & $3.97 \pm 0.42$ & & & & & $98.52 \pm 1.45$ & $19.85 \pm 1.05$ & $9.08 \pm 0.33$ & 75 \\
\hline 'Amol3(sona)' & $7.31 \pm 0.35 * *$ & & & & & & & & \\
\hline CSSL-P271 & $5.48 \pm 0.35^{* *}$ & $q S B 11^{H J X 74}$ & 11 & 0.76 & 19.06 & $98.55 \pm 1.22$ & $19.23 \pm 1.35$ & $8.63 \pm 0.58$ & 74 \\
\hline CSSL-P272 & $5.29 \pm 0.33 * *$ & $q S B 1-1^{H J X 74}$ & 1 & 0.66 & 16.67 & $97.55 \pm 1.86$ & $20.81 \pm 0.95$ & $8.77 \pm 0.81$ & 75 \\
\hline CSSL-P273 & $4.42 \pm 0.30$ & & 1 & & & $97.54 \pm 0.74$ & $20.50 \pm 1.62$ & $8.90 \pm 0.88$ & 75 \\
\hline CSSL-P274 & $3.96 \pm 0.57$ & & 1 & & & $97.78 \pm 1.35$ & $18.45 \pm 0.91$ & $9.37 \pm 0.69$ & 75 \\
\hline CSSL-P275 & $4.01 \pm 0.63$ & & 2 & & & $98.80 \pm 0.53$ & $18.55 \pm 0.89$ & $8.83 \pm 0.63$ & 75 \\
\hline CSSL-P279 & $4.81 \pm 0.32 *$ & $q S B 1-2^{H J X 74}$ & 1 & 0.42 & 10.64 & $97.40 \pm 1.51$ & $19.60 \pm 1.27$ & $8.98 \pm 0.77$ & 75 \\
\hline CSSL-P280 & $4.57 \pm 0.91$ & & 2 & & & $97.74 \pm 1.11$ & $18.95 \pm 0.93$ & $8.73 \pm 0.77$ & 75 \\
\hline CSSL-P281 & $4.30 \pm 0.51$ & & 7 & & & $97.45 \pm 1.71$ & $19.88 \pm 1.40$ & $9.07 \pm 0.60$ & 75 \\
\hline CSSL-P282 & $2.80 \pm 0.90 * *$ & $q S B 2^{A M}$ & 2 & -0.59 & -14.78 & $98.67 \pm 0.95$ & $21.15 \pm 1.49$ & $8.92 \pm 0.38$ & 75 \\
\hline CSSL-P283 & $3.64 \pm 0.79$ & & 9 & & & $98.13 \pm 0.98$ & $19.72 \pm 1.57$ & $8.98 \pm 0.48$ & 75 \\
\hline CSSL-P284 & $3.17 \pm 0.23 *$ & $q S B 3^{A M}$ & 3 & -0.40 & -10.12 & $97.37 \pm 0.70$ & $20.45 \pm 1.40$ & $9.00 \pm 0.59$ & 75 \\
\hline
\end{tabular}

${ }^{\mathrm{z}}$ SBR QTL, SBR quantitative trait loci; PH, plant height; TA, tiller angle; TN, tiller number; HD, heading date. Phenotypic value of SB disease and agronomic traits of all lines are the mean value of 2 years (2007 and 2008) \pm SD, each independent year includes three replications. * and $* *$ indicate $5 \%$ and $1 \%$ significance levels, respectively (two-way ANOVA using Dunnett's method). 
preliminary analysis. Thus, these 11 lines were selected for further evaluation.

Identification of sheath blight resistance QTLs. In 2008, the 11 CSSLs and the two parents were repeatedly evaluated for SB resistance in field and were measured for major morphological traits. Results showed that all CSSLs consistently showed morphological traits similar to the 'HJX74' parent that included PH, TN, HD, TA (Table 1). This characteristic of the 11 CSSLs was crucial for accurate identification of SBR QTLs and evaluation of their effects. The SB ratings of the 13 lines (including parents) showed highly significant correlation with the data in $2007(R=0.835, P=$ 0.000). Based on the SB ratings data from these 2 years (2007 and 2008), the difference in resistance between CSSLs and 'HJX74' was revealed by two-way ANOVA using the Dunnett's method for means separation (Table 1). Three CSSLs (P271, P272, and P279) were found to have significantly higher SB ratings than 'HJX74', indicating that 'HJX74' contained SBR QTLs at the regions containing substituted chromosomal segments in these three CSSLs. Two CSSLs (P282 and P284) showed stronger resistance than 'HJX74', suggesting that the corresponding substituted segments from 'Amol3(sona)' contained putative SBR QTLs. All other lines appeared to have similar levels of resistance to 'HJX74' (Table 1). Based on the chromosomal location of each donor segment in these five CSSLs, we named them QTLs $q S B 1-1^{H J X 74}$ (on the short arm of chromosome 1), qSB1-2 $2^{H J 74}$ (on the long arm of chromosome 1), $q S B 2^{A m}$ (on the long arm of chromosome 2), $q S B 3^{A m}$ (on the long arm of chromosome 3 ), and $q S B 11^{H J X 74}$ (on the long arm of chromosome 11) (Fig. 2).

Through map integration, we found that all five SBR QTLs were in agreement with previous results (Fig. 2). However, $q S B 2^{A m}$ and

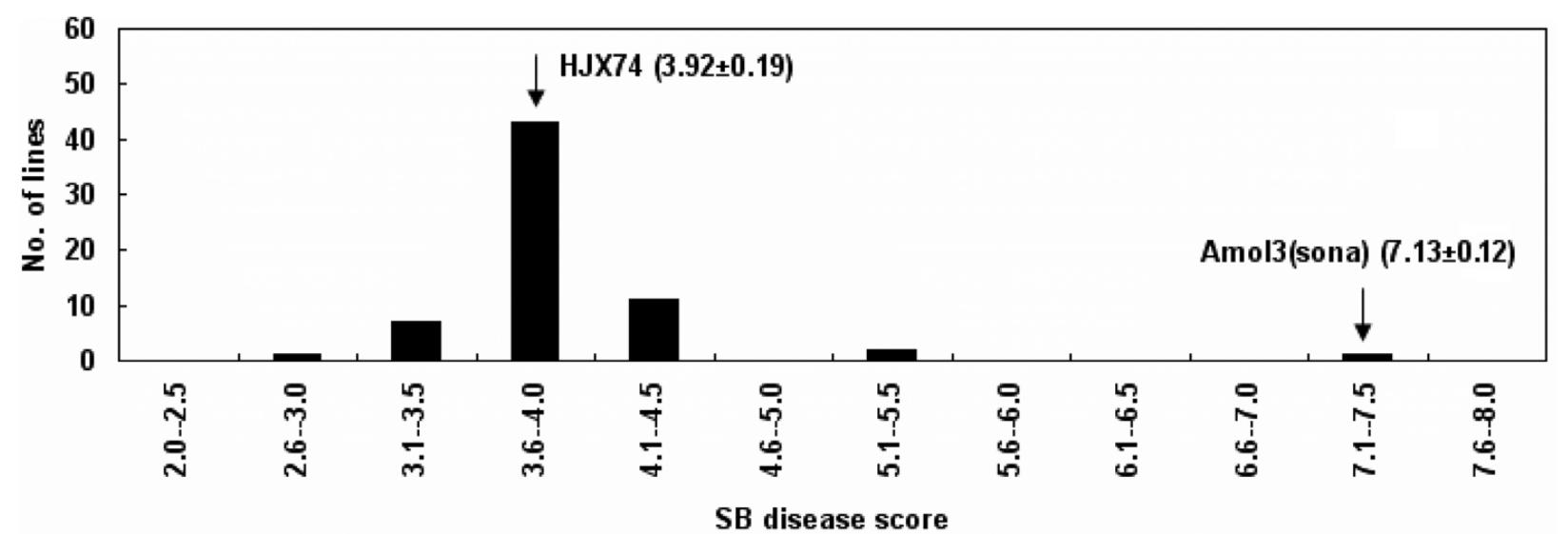

Fig. 1. Histogram distribution diagram of sheath blight (SB) disease scores of chromosome segment substitution lines (CSSLs) and two parents in the 2007 field trial. SB disease scores for all CSSLs ranged from $2.65 \pm 0.25$ to $5.44 \pm 0.11$. Scores of controls 'HJX74' and 'Amol3(sona)' were $3.92 \pm 0.19$ and $7.13 \pm 0.12$, respectively.
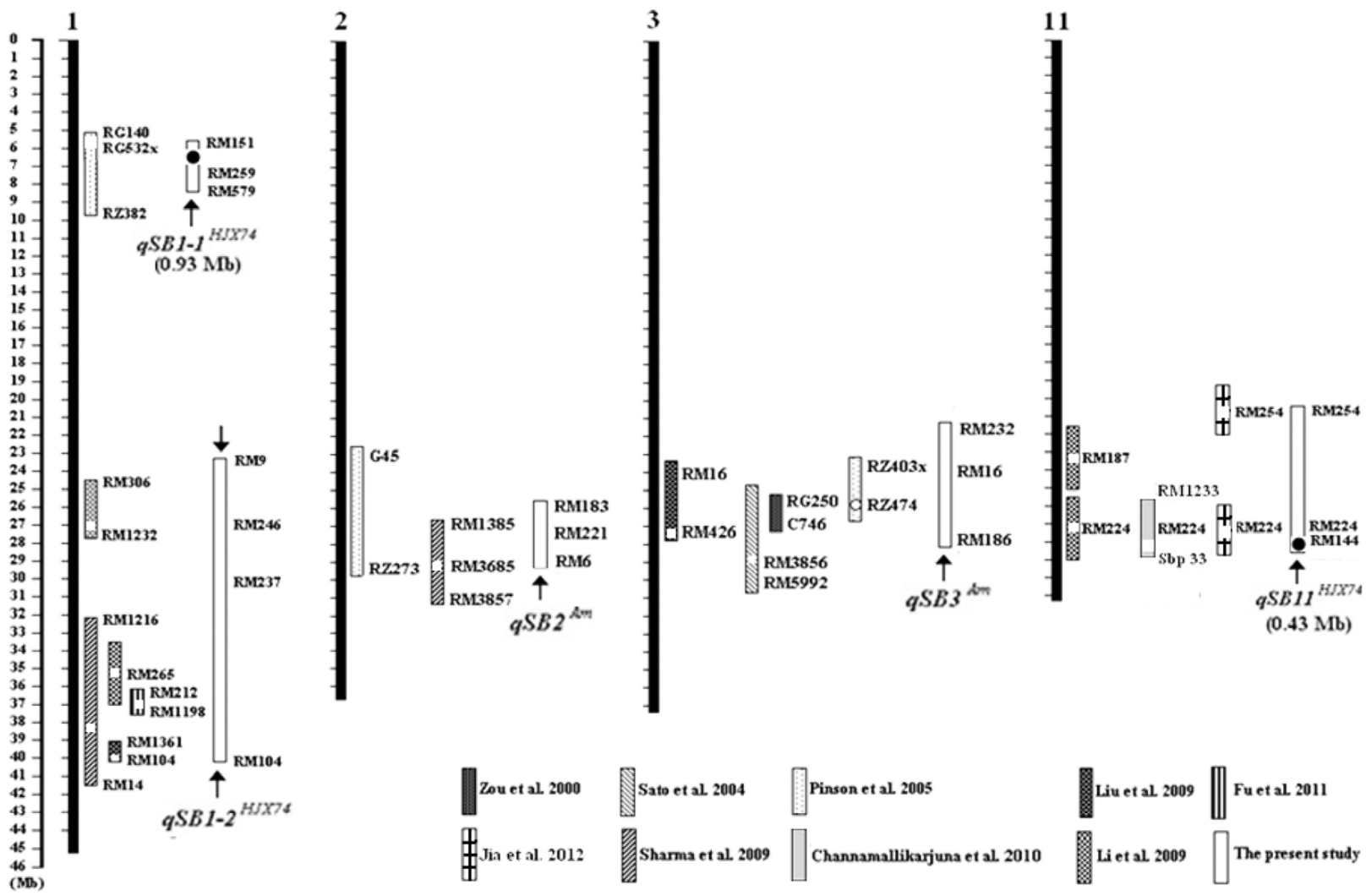

Fig. 2. Locations of sheath blight resistant quantitative trait loci (SBR QTLs) identified in the present study and comparison with previous results. The length of each chromosome was derived from the IRGSP Build5 pseudomolecules of rice variety Nipponbare. Locations of the SBR QTLs identified in this study were estimated according to the positions and length of the introgressed donor chromosomal segment in the chromosome segment substitution line. Physical distances of two fine-mapped SBR QTLs, qSB1-1HJX74 and qSB11 ${ }^{H J X 74}$, are indicated by black circles on the introgressed segments. Previously described SBR QTLs in this figure are from Zuo et al. (48). 
$q S B 3^{A m}$ were found from susceptible varieties, while the previous SBR QTLs at these regions were all from moderately resistant varieties, such as TeQing and Jasmine 85. Although the other three SBR QTLs were all found from moderately resistant varieties, only one of them (on chromosome 11) was confirmed by association analysis (14). Since all CSSLs used in the present study were practically NILs to 'HJX74', we were able to confirm these five SBR QTLs with reliable resistance in the field and their dissociation with plant morphological/developmental traits that was not addressed in the previous studies, including the SBR QTL on chromosome 11. Since $q S B 11^{H J X 74}$ and $q S B 1-1^{H J X 74}$ exhibited greater effects than the other SBR QTLs mentioned above and their resistant alleles were all from the moderately resistant parent 'HJX74' (Table 1, Fig. 3), we selected these SBR QTLs for further analysis to accelerate their utilization.

Genetic modes of $q S B 11^{H J X 74}$ and $q S B 1-1^{H J X 74}$. For determining the genetic modes of inheritance of $q S B 11^{H J X 74}$ and $q S B 1$ $1^{H J X 74}$, we generated two $\mathrm{F}_{1}$ lines by crossing CSSL-P271 and 'HJX74', and CSSL-P272 and 'HJX74', respectively, in 2009 and 2010. The two $F_{1}$ lines, together with 'HJX74', P271 and P272, were then evaluated for resistance to SB. Seven agronomic traits were also measured and recorded (Table 2). The results showed that no significant differences (analyzed by two-way ANOVA and Tukey's method) existed among the five lines in terms of HD, PH $(F=1.599, P=0.218)$, PL $(F=1.090, P=0.391)$, FLL $(F=1.139$, $P=0.370)$, FLW $(F=0.834, P=0.521)$, TA $(F=2.004, P=$ $0.173)$, or TN $(F=0.719, P=0.590)$, indicating that these lines had similar agronomic traits (Fig. 3D). In addition, we also found that the five lines exhibited similar developmental patterns, as determined by visual observation in the field every week from the seeding stage to the maturing stage. Taken together, these results confirm that the five lines had a similar genetic background to 'HJX74'. This information is crucial for evaluating the accurate effects of SBR QTLs.

All five lines were inoculated in the field with a population scale three times the size of that in the previous 2 years. The differences of the average SB rating scores among the lines were revealed by two-way ANOVA. We found that a highly significant difference in $\mathrm{SB}$ rating scores existed among the lines $(F=10.080, P=0.000)$, whereas no difference in SB rating scores was found between years $(F=0.003, P=0.958)$, or between lines and years $(F=1.415, P=$ 0.269). Multiple comparisons showed that lines P271 and HJX74/P271 had similar SBR scores; both were significantly higher than that of 'HJX74' (Table 3). This result indicated that $q S B 11^{H J X 74}$ mainly follows a recessive mode of inheritance. The effect of $q S B 11^{H J X 74}$ was further estimated to reduce the SB disease score by $1.20[\approx(1.4+1.05) / 2]$ in the homozygous condition on the " 0 to $9 "$ rating scale (Table 3 ). For $q S B 1-1^{\text {HJX74 }}$, we found that no significant difference existed between 'HJX74' and HJX74/P272, but their SB rating scores were all significantly lower than that of CSSL-P272, suggesting its dominant mode of inheritance (Table $3)$. Simultaneously, the resistance effect of $q S B 1-1^{H J X 74}$ was evident because this SBR QTL significantly reduced the SB disease score by $0.92[\approx(0.88+0.96) / 2]$ regardless of whether it was in the heterozygous or homozygous condition. In summary, we determined the genetic modes of inheritance for the two major SBR QTLs, and further confirmed the SBR QTLs conferring high resistance to SB in the field.

Further mapping of $q S B 11^{H J X 74}$. To further narrow down the region of $q S B 11^{H J X 74}$, we generated an $\mathrm{F}_{2}$ segregating population from a cross between the NIL P271 and 'HJX74'. After inoculation of plants with $R$. solani as described, we examined the phe-
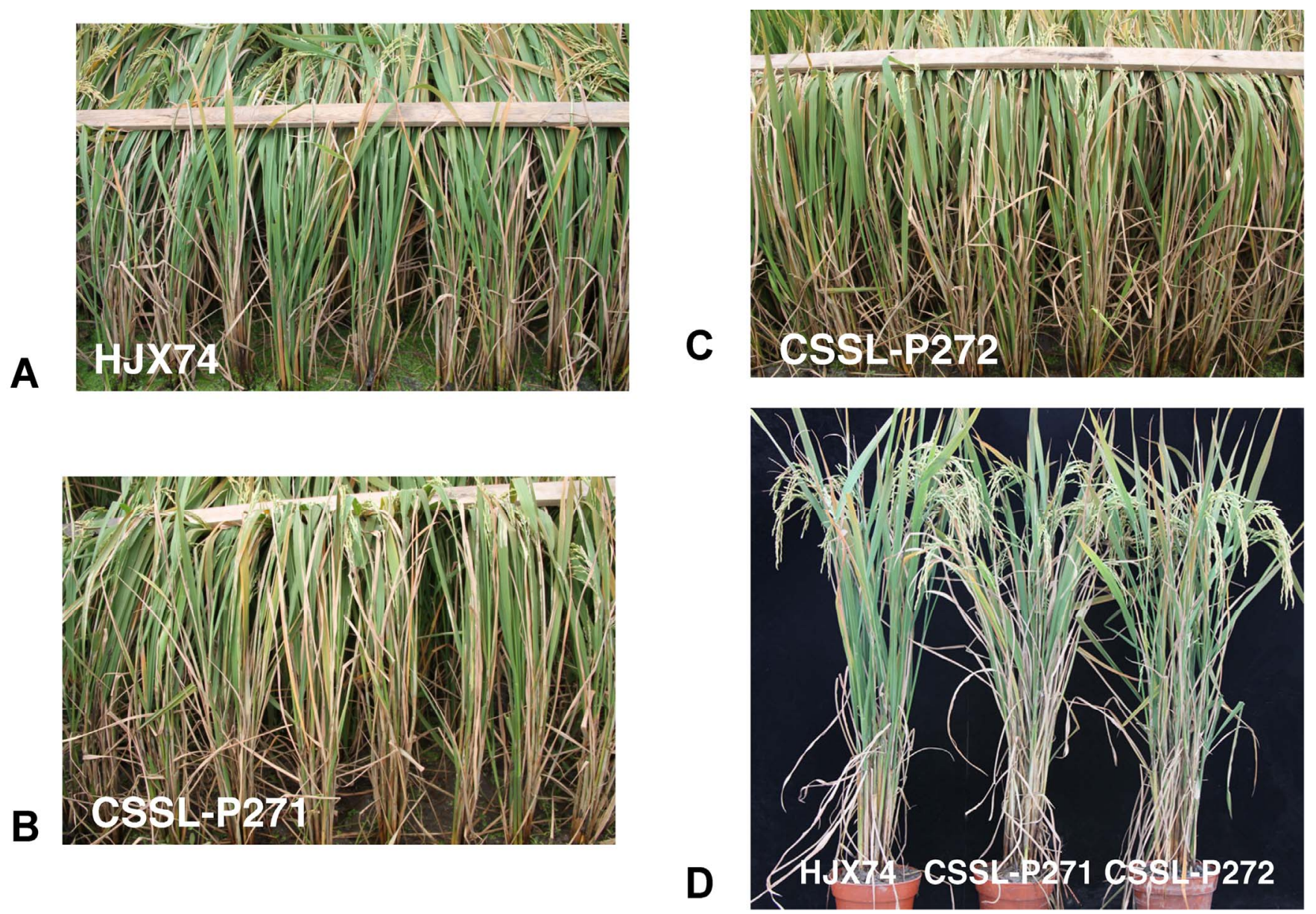

Fig. 3. Resistance performance of rice lines 'HJX74', CSSL-P271 and -P272, at 25 days after heading in the field test. 'HJX74' was the recurrent parent containing major sheath blight resistant quantitative trait loci $q S B 1-1^{H J X 74}$ and $q S B 11^{H J X 74}$; CSSL-P271 and -P272 were the lines without $q S B 11^{H J X 74}$ and $q S B 111^{H J X 74}$, respectively, due to segment substitution in the 'HJX74' background. All lines were inoculated at later tillering stage and pictures were taken at 25 days after heading. 
notypic data for $489 \mathrm{~F}_{2}$ individuals. Based on information about the donor segment (from markers PSM418 to RM144) in CSSLP271, we developed seven new polymorphic markers for genotyping these $\mathrm{F}_{2}$ individuals (Table 4). Two markers (ZY25.9-11-3 and ZY28.23-11-3) were developed to replace PSM418 and RM144, respectively, to increase the efficiency of PCR. Markers ZY24.6-11-4 and ZY28.3-11-5 are located outside PSM418 and RM144, respectively, and the other three markers are located in the interval between PSM418 and RM144. According to a chi-square test (Table 5), all seven markers were found to match the 1:2:1 segregation ratio in the segregating population. The order of the markers on the genetic map is the same as that on the physical map of 'Nipponbare', and the ratio of physical to genetic distance is $291.7 \mathrm{~kb}$ per $\mathrm{cM}$.

The phenotypic data from each $\mathrm{F}_{2}$ plant and the genotypes of the loci represented by the seven markers on each $F_{2}$ plant were then

Table 2. Analysis of agronomic traits among lines 'HJX74', two chromosome segment substitution lines (CSSLs), and two types of $\mathrm{F}_{1}$ lines obtained by crossing each CSSL with 'HJX74'z

\begin{tabular}{lccccccc}
\hline & \multicolumn{7}{c}{ Trait } \\
\cline { 2 - 7 } Line & HD $($ day) & PH $(\mathbf{c m})$ & PL $(\mathbf{c m})$ & FLL $(\mathbf{c m})$ & FLW $(\mathbf{c m})$ & TN & TA $\left({ }^{\circ}\right)$ \\
\hline 'HJX74' & $75.0 \mathrm{a}$ & $98.40 \pm 1.20 \mathrm{a}$ & $27.34 \pm 1.00 \mathrm{a}$ & $37.31 \pm 1.44 \mathrm{a}$ & $1.95 \pm 0.03 \mathrm{a}$ & $8.77 \pm 0.39 \mathrm{a}$ & $20.03 \pm 1.60 \mathrm{a}$ \\
P271 & $74.0 \mathrm{a}$ & $97.07 \pm 0.93 \mathrm{a}$ & $26.95 \pm 0.95 \mathrm{a}$ & $37.65 \pm 0.97 \mathrm{a}$ & $1.97 \pm 0.04 \mathrm{a}$ & $8.73 \pm 0.71 \mathrm{a}$ & $20.33 \pm 1.23 \mathrm{a}$ \\
P272 & $75.0 \mathrm{a}$ & $97.64 \pm 0.98 \mathrm{a}$ & $26.65 \pm 1.11 \mathrm{a}$ & $38.22 \pm 2.10 \mathrm{a}$ & $1.97 \pm 0.05 \mathrm{a}$ & $8.90 \pm 0.92 \mathrm{a}$ & $19.33 \pm 1.15 \mathrm{a}$ \\
$\mathrm{F}_{1}$ (HJX74/P271) & $75.0 \mathrm{a}$ & $97.46 \pm 2.29 \mathrm{a}$ & $26.77 \pm 0.95 \mathrm{a}$ & $37.33 \pm 1.48 \mathrm{a}$ & $1.95 \pm 0.05 \mathrm{a}$ & $9.27 \pm 0.72 \mathrm{a}$ & $20.50 \pm 1.11 \mathrm{a}$ \\
$\mathrm{F}_{1}$ (HJX74/P272) & $75.0 \mathrm{a}$ & $98.93 \pm 0.50 \mathrm{a}$ & $26.43 \pm 0.34 \mathrm{a}$ & $36.74 \pm 0.85 \mathrm{a}$ & $1.93 \pm 0.04 \mathrm{a}$ & $8.97 \pm 0.43 \mathrm{a}$ & $19.28 \pm 0.83 \mathrm{a}$ \\
\hline
\end{tabular}

${ }^{\mathrm{z}} \mathrm{HD}$, heading date; PH, plant height; PL, panicle length; FLL, flag leaf length; FLW, flag leaf width; TN, tiller number; TA, tiller angle. The letter "a" indicates no difference of tested traits among lines at 5\% significance level. Data are the mean value of 2 years (2009 and 2010) \pm SD. Each independent year includes three replications (two-way ANOVA using Tukey's method).

Table 3. Multi-comparison of average sheath blight (SB) disease scores among lines 'HJX74', two chromosome segment substitution lines (CSSLs), and two types of $\mathrm{F}_{1}$ lines obtained by crossing each CSSL with 'HJX74'

\begin{tabular}{lccc}
\hline SB-QTLs & Rice line (genotype of the target SBR QTL) & ${\text { Average SB disease score } \pm \text { SD }^{\mathbf{y}}}^{\text {Estimated effect }^{\mathbf{z}}}$ \\
\hline$q S B 11^{H J X 74}$ & $\mathrm{P} 271\left(q S B 11^{A m}\right)$ & $6.57 \pm 0.49 \mathrm{~A}$ & \\
& HJX74/P271 $\left(q S B 11^{H}\right)$ & $6.23 \pm 0.59 \mathrm{~A}$ & \\
& HJX74 $\left(q S B 11^{H J X 74}\right)$ & $5.17 \pm 0.40 \mathrm{~B}$ & 1.40 \\
& P272 $\left(q S B 1-1^{A m}\right)$ & $6.13 \pm 0.42 \mathrm{~A}$ & 0.88 \\
& HJX74/P272 $\left(q S B 1-1^{H}\right)$ & $5.25 \pm 0.56 \mathrm{~B}$ & 0.96 \\
\hline
\end{tabular}

x SBR QTL, sheath blight resistance quantitative trait loci. HJX74/P271 and HJX74/P272 represent $\mathrm{F}_{1}$ plants obtained by crossing 'HJX74' with P271 and 'HJX74' with P272, respectively. The superscript letters ${ }^{A m},{ }^{H}$, and ${ }^{H J X 74}$ represent the genotypes of homozygous 'Amol3(sola)', the heterozygote, and homozygous 'HJX74', respectively.

${ }^{y}$ Data are mean values of 2 years (2009 and 2010) \pm SD. Each independent year includes three replications. Capital letters A and B following the SBR score indicate $1 \%$ significant difference level (two-way ANOVA using Tukey's method).

${ }^{\mathrm{z}}$ Resistance effect of $q S B 11^{H J X 74}$ was estimated using the average SB disease score of CSSL minus that of 'HJX74'; resistance effect of $q S B 1-1^{H J X 74}$ was the mean of CSSL-P272 minus that of 'HJX74' and HJX74/P272, respectively.

Table 4. Primer sequences and physical positions of molecular markers developed in this study

\begin{tabular}{|c|c|c|c|c|}
\hline \multirow[b]{2}{*}{ Molecular marker ${ }^{\mathrm{y}}$} & \multirow[b]{2}{*}{ Chromosome } & \multirow{2}{*}{$\begin{array}{l}\text { Physical position } \\
(\text { (bp) }\end{array}$} & \multicolumn{2}{|c|}{ Sequences $\left(5^{\prime}-3^{\prime}\right)$} \\
\hline & & & Forward & Reverse \\
\hline ZY24.6-11-4 & 11 & 24699281 & CGAAGGTTGCCCATGTATTT & CCCGTTGTACCGAAGAAGAG \\
\hline ZY25.9-11-3 & 11 & 25966392 & AACTCTTGTAACAAAAAGGT & GCAAAGTGCAAATAATCATC \\
\hline ZY26.6-11-2 & 11 & 26511355 & GGTAATTTAACTATCTTTG & TATCGTATCTTTTCTCTTC \\
\hline ZY27.49-11 & 11 & 27526408 & GTTTGTCTGCGATGCCGATTC & GCCTTTGCCCGAGACCCTA \\
\hline ZY27.92-11 & 11 & 27960085 & TCGAACTCAAGATGCAACA & ACATACCGTGAAACAAACA \\
\hline ZY28.23-11-3 & 11 & 28272062 & AGGGCACAGAGGGAACAAT & ACAGGGTCAGGCAGTCAGG \\
\hline ZY28.3-11-5 & 11 & 28414696 & GTGCGAAGTACAAGGAAGC & CCGCCAACTACGACCCTAC \\
\hline ZY5.2-1-1 & 1 & 5151106 & GCGTAGATCCGGTCAGCTC & AGTCCCTCGCAACCAAATG \\
\hline ZY6.9Mb-1-9 & 1 & 7005357 & CAGCCTCGTGTTTTCGTTA & TTTTGGTTGCATATTAGCG \\
\hline ZY7.1Mb-1-4 & 1 & 7154368 & TGTGGGTGTCGATCTAGGAG & GTAGCCAGGGAAGAGGTGTT \\
\hline ZY7.7Mb-1-5 & 1 & 7607617 & ACTCAAATATACTCCCTTTCGT & TTGTTGTTACTTTCTTCGTGCT \\
\hline
\end{tabular}

y $\mathrm{ZY}$ indicates the InDel (Insertion/Deletion) markers designed with polymorphisms between 'HJX74' and 'Amol3(sona)'.

${ }^{\mathrm{z}}$ Physical position on the IRGSP Build5 pseudomolecules of rice variety Nipponbare.

Table 5. Segregation ratio of markers in the mapping population for $q S B-11^{H J X 74}$

\begin{tabular}{lccccc}
\hline Molecular marker & $\begin{array}{c}\text { Recipient parent } \\
\text { genotype }\end{array}$ & $\begin{array}{c}\text { Heterozygous } \\
\text { genotype }\end{array}$ & $\begin{array}{c}\text { Donor parent } \\
\text { genotype }\end{array}$ & $\boldsymbol{\chi}^{\mathbf{2}(1: 2: 1)}$ & $\boldsymbol{P}_{\text {value }}^{\mathbf{z}}$ \\
\hline ZY25.9-11-3 & 123 & 238 & 126 & 0.285 & $0.867 \mathrm{a}$ \\
ZY26.6-11-2 & 120 & 246 & 119 & 0.105 & $0.949 \mathrm{a}$ \\
RM224 & 119 & 248 & 121 & 0.148 & $0.929 \mathrm{a}$ \\
ZY27.49-11 & 118 & 246 & 124 & 0.180 & $0.914 \mathrm{a}$ \\
ZY27.92-11 & 117 & 246 & 124 & 0.253 & $0.881 \mathrm{a}$ \\
ZY28.23-11-3 & 117 & 251 & 121 & 0.411 & $0.814 \mathrm{a}$ \\
ZY28.3-11-5 & 117 & 249 & 119 & 0.365 & $0.833 \mathrm{a}$ \\
\hline
\end{tabular}

${ }^{\mathrm{z}}$ The letter "a" indicates no difference between the segregation ratio of the markers and the expected segregation ratio (1:2:1) at 5\% significance level. 
used to perform QTL mapping by CIM. The results indicate that $q S B 11^{H J X 74}$ is most likely located in the region of ZY27.49 and ZY27.92-11, with the highest LOD value at 9.2 (Fig. 4A). This region covers approximately $430 \mathrm{~kb}$ on the IRGSP Build5 pseudomolecules of rice variety Nipponbare. In this experiment, $q S B 11^{H J X 74}$ could explain $12.08 \%$ of the total phenotypic variation, with an additive effect of -0.76 . As shown in Figure 3, we found that marker ZY27.92-11 is very close to the LOD peak. We then classified all $F_{2}$ plants into three groups: homozygous 'HJX74', homozygous 'Amol3(sona)', and heterozygous based on the genotype of the ZY27.92-11 locus. According to the frequency distribution of plants on SB disease ratings from 3.0 to 8.5 (Fig. 4B), we found that plants with homozygous 'HJX74' genotype at ZY27.9211 showed clearly lower SB disease scores (range from 3.0 to 6.5) compared to plants with homozygous 'Amol3(sona)' genotype (from 4.0 to 8.5). These results further confirm the existence of $q S B 11^{H J X 74}$ and its linkage with molecular marker ZY27.92-11.

Further mapping of $q S B 1-1^{H J X 74}$. To further map $q S B 1-1^{H J X 74}$, two CSSLs, P272 and P274 were used (Table 4). The two CSSLs had overlapping, introgressed segments but showed highly significant difference in resistance as revealed by ANOVA using SB dis- ease scores from each year (one-way ANOVA) and from 3 years (two-way ANOVA) (Fig. 5). The average SB disease score of the 3year data of CSSL-P272 (5.37 \pm 0.36$)$ was significantly higher than those of recurrent parent 'HJX74' $(4.03 \pm 0.47)$ and CSSLP274 (4.02 \pm 0.49$)$ (Fig. 5); while there was no significant difference between 'HJX74' and CSSL-P274. Therefore, the CSSL-P272 and CSSL-P274 were defined as susceptible and resistant phenotypes, respectively, for subsequent mapping. In addition, the two CSSLs both showed significantly higher resistance than the susceptible parent 'Amo13(sona)', which gave an average SB disease score of $7.39 \pm 0.37$.

Five new polymorphic markers were developed to identify the genotype on the region of substituted segment of CSSL-P272 and P274. Through genotype comparison, the susceptible introgressed segments of the two CSSLs overlapped at markers RM151 and RM490 (Fig. 5). Since on the left side of RM151, the susceptible introgressed segment in resistant CSSL-P274 was longer than that in susceptible CSSL-272, the $q S B 1-1^{H J X 74}$ QTL should locate on the right side of RM490, but not on the left side of RM151. On the right side of RM490, three new markers were identified with susceptible 'Amol3(sona)' genotype in the susceptible CSSL-P272,
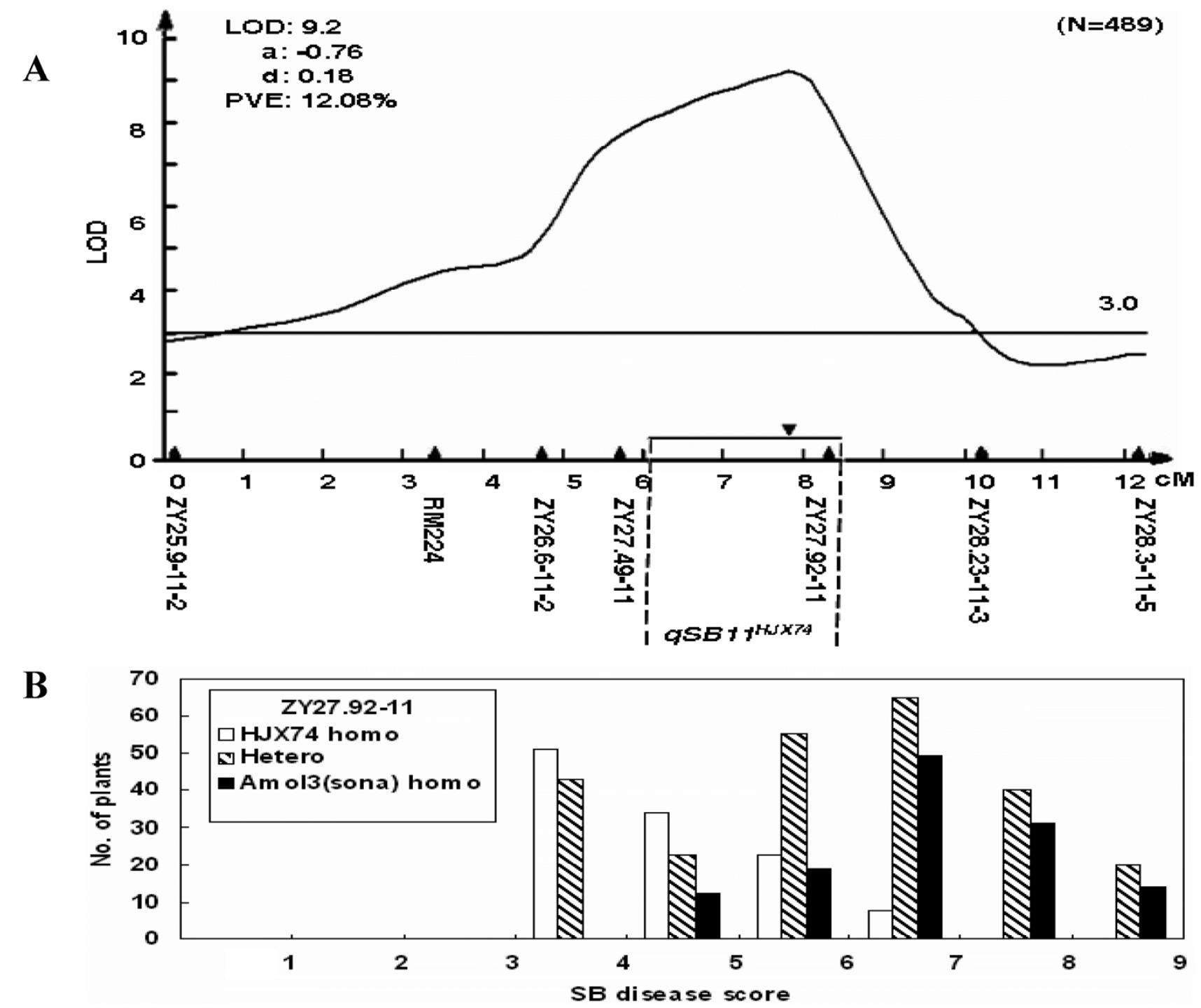

Fig. 4. Chromosomal location of the quantitative trait loci (QTL) on the long arm of chromosome 11. A, Molecular markers are indicated under the $x$-axis. The long horizontal line indicates the threshold value of 3.0 for determining a putative QTL on chromosome 11 in the $F_{2}$ population of HJX74 $\times$ CSSL-P271 (489 plants). The short horizontal line and the arrow on the line indicate the confidence interval of $q S B 11^{H J X 74}$ and the logarithm of odds (LOD) peak, respectively. LOD, a, d, and PVE indicate peak value of logarithm of odds, additive effect, dominance effect, and phenotypic variation explained, respectively. B, Frequency distribution of $F_{2}$ plants on sheath blight (SB) disease scores. Three types of $\mathrm{F}_{2}$ plants were classified according to their genotypes at marker ZY27.92-11 locus that identified close to LOD peak. White, hatched, and black columns indicate homozygous 'HJX74' genotype, heterozygous genotype, and homozygous 'Amol3(sona)' genotype. SB disease score of each $\mathrm{F}_{2}$ plant was investigated at 30 days after heading. 
which contained resistant 'HJX74' genotypes for markers ZY7.71-5 and RM579. Therefore, the ZY7.7-1-5 marker should be the nearest right border of $q S B 1-1^{H J X 74}$. Because no more polymorphic markers were developed between RM490 and ZY6.9-1-9, or between RM259 and ZY7.7-1-5, the $q S B 1-1^{H J X 74}$ was finally physically narrowed down to the region between markers RM490 and ZY7.7-1-5, covering approximately $930 \mathrm{~kb}$ on the reference genome of the japonica rice variety Nipponbare (Fig. 5).

\section{Discussion}

Comparison of co-located SBR QTLs. Based on the integrated map of SBR QTLs published in 2010, Zuo et al. (46) has found that many SBR QTLs located in the same or overlapping chromosomal regions showing various resistance effects. For example, in the chromosomal region that contains $q S B-9^{T Q}$, SBR QTLs from rice varieties Pecos (PE), Jasmine 85 (J85), and MCR10277 (MCR) have also been identified (45). Among these, $q S B-9^{M C R}$ could explain up to $34 \%$ of phenotypic difference (27), which was apparently higher than those of $q S B-9^{J 85}$ (45) and $q S B-9^{P E}$ (32). However, at the present time we cannot determine whether these co-located SBR QTLs are controlled by the same gene or not.

In the present study, we identified five SBR QTLs, which were all in agreement with previous results about the presence of SBR QTLs in these regions (Fig. 2). However, although $q S B 1-1^{H J X 74}$ is located in the same region as $q S B-1^{T Q}$ (30), these two QTLs contribute differently to $\mathrm{SB}$ resistance. Specifically, $q S B 1-1^{H J X 74}$ reduced SB disease scores by approximately 1.3 on the 0 to 9 rating scale and explained approximately $16.7 \%$ of the total phenotypic difference (Table 1), which was higher than the $8 \%$ contributed by $q S B-1^{T Q}$ (30). For $q S B 2^{A m}$ and $q S B 3^{A m}$, although their resistance effects were found no obvious difference compared to the previous co-located SBR QTLs, their resistant alleles come from a completely susceptible variety 'Amol3(sona)' that was totally different from the previous studies $(22,30,32,45)$. This implies various mechanisms may exist between these two SBR QTLs and their co-located SBR QTLs. $q S B 11^{H J X 74}$ is co-localized with three other reported SBR QTLs, but with different contributions to SB resistance $(4,14,19)$. Among them, the qSBR11-1 QTL, detected on the well-known SB resistant variety Tetep (4), has been mapped into an $850 \mathrm{~kb}$ region that includes $q S B 11^{H J X 74}$. Moreover, $q S B R 11-1$ showed similar effect to $q S B 11^{H J X 74}$. Thus, they are likely controlled by the same allele. Through association mapping, Jia et al.(14) found a few SB loci with numerous alleles that showed different contributions to SB resistance. However, nearly all co-located SBR QTLs were mapped in a region more than $1,000 \mathrm{~kb}$ in length except for the co-located qSBR11-1 and $q S B 11^{H J X 74}$ QTLs. Therefore, we cannot determine if these colocated SBR QTLs are controlled by allelic genes or different genes at the present time, especially for those with different resistant effects.

Utilization and further mapping of SBR QTLs. In previous studies, many SBR QTLs were found to co-localize with QTLs controlling $\mathrm{HD}$ and a few plant morphological traits $(10,21,30,43,46)$. This is expected because rice SB resistance is easily influenced by microenvironment that can be greatly affected by plant morphology, especially HD, PH, TA, and TN. Therefore, the SBR QTLs co-located with these morphological QTLs are likely "ghost" SBR QTLs. Certainly, there is also the possibility that these two types of QTLs are linked together. However, in both cases, their breeding value will be greatly reduced (10). To eliminate these circumstances, we first excluded 13 CSSLs, which showed difference on these morphological and developmental traits compared with the control 'HJX74', and used the remaining 50 CSSLs for preliminary SB resistance analysis. Out of the 50 CSSLs, 11 candidates were repeatedly evaluated in field. We found all 11 CSSLs showed consistent morphological/developmental traits as 'HJX74' (Table 1), which is of crucial importance for accurately identifying the effect of SBR QTLs. Through a 2-year evaluation test, we finally identified five SBR QTLs that all showed significant effects on resistance in the field (Table 1). A few lines, for example P273, P280, and P283, showed either a lower or higher SB disease score than that of 'HJX74' but not reaching statistically significant levels (Table 1). The slight differences between these lines and 'HJX74' may be attributed to experimental errors, rather than to QTLs. Certainly, we cannot exclude the possibility that these lines actually contain weaker SB resistance QTLs. However the resolution in the present field evaluation system was not high enough to identify these weaker QTLs. Two of the five SBR QTLs were further confirmed by evaluations in the field trials in another 2 years, which indicated the high reliability of the five SBR QTLs identified in the trials of the first 2 years (Table 3). In all evaluations, the late tillering stage and approximately 30 days after heading were set as the inoculation and disease rating time points, respectively, which capture the most serious stage of SB development in rice (51). Consequently, the five SBR QTLs reported here are more reliable and useful in breeding practice compared to their co-located SBR QTLs in previous studies.

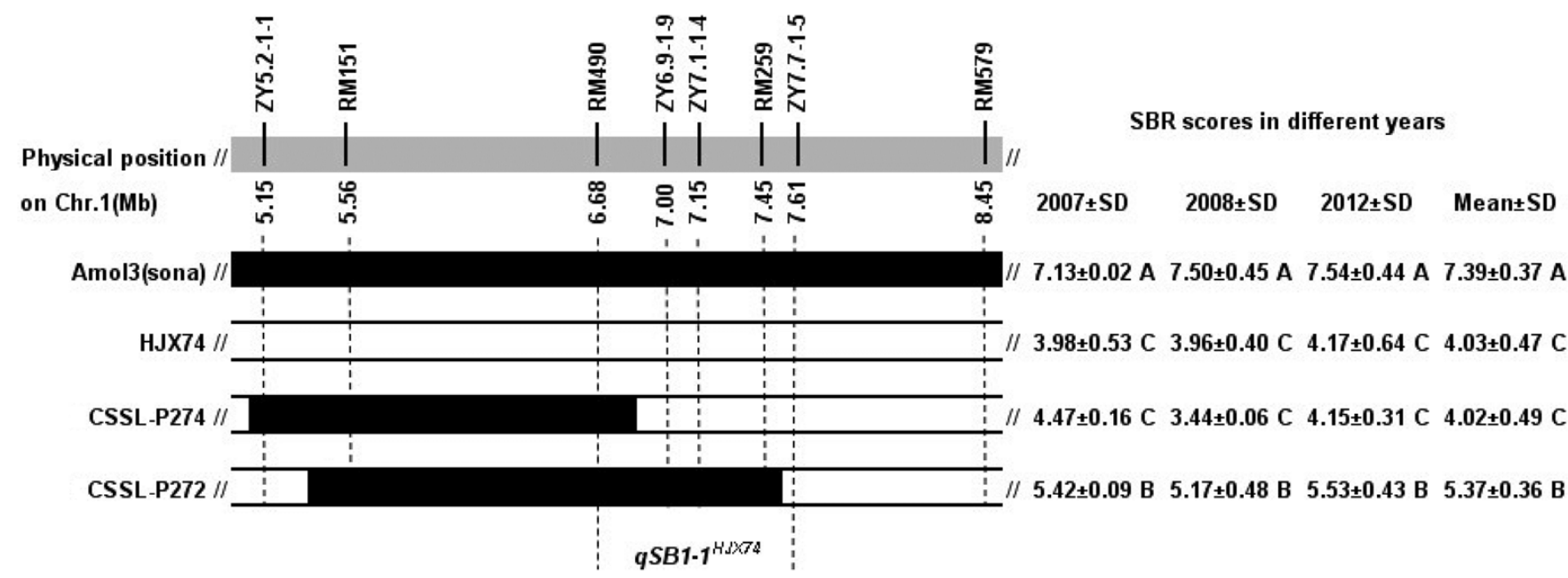

Fig. 5. Physical map of $q S B 1-1^{H J X 74}$ on chromosome 1. Gray bar represents rice chromosome 1 (Chr. 1). Molecular markers and their physical positions (on the IRGSP Build5 pseudomolecules of rice variety Nipponbare) are indicated on Chr. 1. Black and white bars represent chromosomal segments of Chr. 1 from the donor (parent Amo13[sona]) and the recurrent parent ('HJX74'), respectively. The right panel shows the difference on the sheath blight resistance score of four lines in different years (oneway ANOVA using Tukey's method), and the average scores of 3 years (two-way ANOVA using Tukey's method). Phenotypic data of each line in each year were represented by the average value of three replications. Each replication in 2007 and 2008 included 10 inoculated plants, but in 2012 consisted of 30 inoculated plants. Capital letters following the average sheath blight disease score indicate 1\% significant difference level. The qSB1-1HJX74 is located between RM490 and ZY7.7-1-5. 
In order to accelerate the utilization of major SBR QTLs, we further investigated the genetic modes of the two SBR QTLs with the most significant effects out of five QTLs. Genetic mode of inheritance is one of the important factors in breeding practice and is required for making a specific breeding plan (28). Previous studies have found that regardless of whether $q S B-9^{T Q}$ was in the homozygous or heterozygous state, this QTL exhibits a similar resistance effect in the field, while $q S B-11^{L E}$ acts in a dosagedependent manner $(36,47,49)$. This result implies that for hybrid rice breeding, introducing $q S B-9^{T Q}$ into one of the parents would be sufficient to allow the hybrid rice to obtain maximal resistance from $q S B-9^{T Q}$. However, introducing $q S B-11^{L E}$ into both hybrid parents would be required to obtain the maximal benefit in a hybrid rice line. In the present study, we carefully evaluated the resistant effects of $q S B 1-1^{H J X 7}$ and $q S B 11^{H J X 74}$ in heterozygous condition by using $F_{1}$ lines constructed between each of two CSSLs and 'HJX74'. Through comparison among three lines with different genotypes, we found that $q S B 1-1^{H J X 74}$, like $q S B-9^{T Q}$, functioned as a dominant resistance QTL, whereas $q S B 11^{H J X 74}$ acted in a recessive manner (Table 3). These results indicate that different strategies need to be employed in making a breeding program using these two SBR QTLs, because $q S B 11^{H J X 74}$, but not $q S B 1-1^{H J X 74}$, has none or only weak resistance effect in the heterozygous status.

To increase the efficiency of MAS, we further narrowed down the regions of the two major SBR QTLs. We fortunately found two CSSLs for $q S B 1-1^{H J X 74}$ that possessed overlapping introgressed segments but showed significant differences in SB resistance (Fig. 5). In addition, the introgressed segment in one of the CSSLs began at the short-arm end of chromosome 1 . These data allowed us to determine the physical position of $q S B 1-1^{H J X 74}$ by comparing the lengths of the introgressed segments in the two CSSLs (Fig. 5). By developing new polymorphic markers, we successfully finemapped $q S B 1-1^{H J X 74}$ to a $930-\mathrm{kb}$ region. We adopted the traditional QTL mapping method for $q S B 11^{H J X 74}$ to identify its most likely region. This technique had been successfully used to narrow down a major SBR QTL, $q S B R 11-1$, by Channamallikarjuna et al. (4). All $\mathrm{F}_{2}$ individuals in the $q S B 11^{H J X 74}$ mapping population had the same genetic background. Therefore, the influence of plant architecture on disease development was eliminated, which greatly increased the reliability of the phenotypic data used in the QTL mapping. Through composite interval mapping, the $q S B 11^{H J X 74}$ was further mapped into a confidence interval covering approximately $430 \mathrm{~kb}$ (Fig. 4A). By using marker ZY27.92-11, closest to the LOD peak, we found that all plants with the homozygous 'HJX74' genotype at this marker locus showed lower SB disease scores than those with the homozygous 'Amol3(sona)' genotype (Fig. 4B). Therefore, the ZY27.92-11 marker is an efficient one for selecting $q S B 11^{H J X 74}$ and can be directly used in breeding programs. In addition, if $q S B 11^{H J X 74}$ is controlled by the same allele as $q S B R 11-1$ (see the discussion in above), we would in effect have further mapped this major QTL into a smaller region. So far, only one SBR QTL, $q S B-11^{L E}$, was fine-mapped (47). Nearly all other SBR QTLs were mapped in regions with an average length of $3.64 \mathrm{Mb}$ (50). Therefore, the accomplishment in mapping of $q S B 11^{H J X 74}$ in this study is a very significant step toward its cloning.

The indica variety 'HJX74' is one of the well-known breeding parents in southern China that has been widely used in both hybrid rice breeding and inbred rice breeding (1). SB disease has become the most destructive rice disease in southern China. Therefore, the three identified SBR QTLs from 'HJX74' are of high breeding potential as they can be employed soon in a breeding program against $\mathrm{SB}$ in this region. In addition, in rice sheath blight resistance, there also have been some SBR QTLs identified from relatively susceptible varieties $(30,47,50)$. The resistant alleles from susceptible parents have also been reported in many studies of resistance to other diseases that have been considered a rare valuable resource for producing super resistant variety $(29,42)$. In the present study, two SBR QTLs identified from the susceptible variety 'Amol3(sona)' are therefore of special breeding value in developing varieties with high resistance to SB.

\section{Acknowledgments}

We thank Scot Nelson and Mawsheng Chern for their helpful suggestions in improving the manuscript and for English editing. This work was partially supported by grants from Jiangsu Agriculture Science and Technology Innovation Fund (CX(12)1003), Jiangsu Science \& Technology Pillar Program (BE2012303), the Project Funded by the Priority Academic Program Development of Jiangsu Higher Education Institutions (PAPD), and the Innovation Project of Jiangsu Province for Graduate Student (CXLX12_0923).

\section{Literature Cited}

1. Akshay, T., and Zhang, Q. G. 2003. Development of single segment substitution lines (SSSLs) and mapping of QTLs in rice (Oryza sativa L.). Mol. Plant Breed. 14:565-567.

2. Bernardes-de-Assis, J., Storari, M., Zala, M., Wang, W. X., Jiang, D. H., Dong, L. SH., Jin, M. S., McDonald, B. A., and Ceresini, P. C. 2009. Genetic structure of populations of the rice-infecting pathogen Rhizoctonia solani AG-1 IA from China. Phytopathology 99:1090-1099.

3. Brooks, S. 2007. Sensitivity to a phytotoxin from Rhizoctonia solani correlates with sheath blight susceptibility in rice. Phytopathology 97:12071212 .

4. Channamallikarjuna, V., Sonah, H., Prasad, M., Rao, G. J. N., Chand, S., Upreti, H. C., Singh, N. K., and Sharma, T. R. 2010. Identification of major quantitative trait loci qSBR11-1 for sheath blight resistance in rice. Mol. Breed. 25:155-166.

5. Chen, G. B., Zhang, F. T., Zhu, Z. X., and Zhu, J. 2011. QGAStation 2.0. Institute of Bioinformatics of Zhejiang University, China. Retrieved 1 July 2011 from http://ibi.zju.edu.cn/software/qga/v2.0/index.htm

6. Chen, Z. X., Zuo, S. M., Zhang, Y. F., Zhu, J. K., Wang, L. P., Feng, F., Ma Y. Y., and Pan, X. B. 2012. Rice pyramiding breeding using sheath blight resistance QTL $q S B-9^{T Q}$ and stripe disease resistance gene $S t v-b^{i}$. Acta Agron. Sin. 38(7):1-10

7. Eizenga, G. C., Agrama, H. A., Lee, F. N., and Jia, Y. 2009. Exploring genetic diversity and potential novel disease resistance genes in a collection of rice (Oryza spp.) wild relatives. Genet. Resour. Crop Evol. 56:65-76.

8. Eshed, Y., and Zamir, D. 1995. An introgression line population of Lycopersicon pennellii in the cultivated tomato enables the identification and fine mapping of yield-associated QTL. Genetics 141:1147-1162.

9. Fu, D., Chen, L., Yu, G. H., Liu, Y., Lou, Q. J., Mei, H. W., Xiong, L., Li, M. S., Xu, X. Y., and Luo, L. J. 2011. QTL mapping of sheath blight resistance in a deep-water rice cultivar. Euphytica 2:209-218.

10. Han, Y. P., Xing, Y. Z., Gu, S. L., Chen, Z. X., Pan, X. B., and Chen, X. L. 2003. Effect of morphological traits on sheath blight resistance in rice. Acta Botanica Sin. 7:825-831.

11. Hao, W., Zhu, M. Zh., Gao, Ji. P., Sun, Sh. Y., and Lin, H. X. 2009. Identification of quantitative trait loci for rice quality in a population of chromosome segment substitution lines. J. Integr. Plant Biol. 51(5):500-512.

12. Huang, S. W., Wang, L., Huang, W. W., and Liu, L. M. 2009. Prevention and control technology theory and practice to the main diseases and insect pests of rice. China Rice 4:13-16.

13. Ji, Q., Lu, J. F., Chao, Q., Gu, M. H., and Xu, M. L. 2005. Delimiting a rice wide-compatibility gene $\mathrm{S} 5 \mathrm{n}$ to a $50 \mathrm{~Kb}$ region. Theor. Appl. Genet. 111:1495-1503.

14. Jia, L. M., Yan, W. G., Zhu, C. S., Agram, A. H., Jackson, A., Yeater, K., Li, X. B., Huang, B. H., Hu, B. L., McClung, A., and Wu, D. X. 2012. Allelic analysis of sheath blight resistance with association mapping in rice. PLoS ONE. 7(3):e32703.

15. Jia, Y. L., Correa-Victoria, F., McClung, A., Zhu, L., Liu, G. J., Wamishe, Y., Xie, J., Marchetti, M. A., Pinson, S. R. M., Rutger, J. N., and Correll, J. C. 2007. Rapid determination of rice cultivar responses to the sheath blight pathogen Rhizoctonia solani using a micro-chamber screening method. Plant Dis. 91:485-489.

16. Jia, Y. L., Liu, G. J., Costanzo, S., Lee, S. H., and Dai, Y. T. 2009. Current progress on genetic interactions of rice with rice blast and sheath blight fungi. Front Agric. China 3:231-239.

17. Kubo, T., Nakamura, K., and Yoshimura, A. 1999. Development of a series of Indica chromosome segment substitution lines in Japonica background of rice. Rice Genet. Newsl. 16:104-106.

18. Lee, F. N., and Rush, M. C. 1983. Rice sheath blight: A major rice disease. Plant Dis. 67:829-832.

19. Li, F., Cheng, L. R., Xu, M. R., Zhou, Z. H., Zhang, F., Sun, Y., Zhou, Y. L., Zhu, L. H., Xu, J. L., and Li, Z. K. 2009. QTL mining for sheath blight resistance using the backcross selected introgression lines for grain quality in rice. Acta Agron. Sin. 9:1729-1737.

20. Li, Sh. G., Cui, G. K., Guan, Ch. R., Wang, J., and Liang, G. H. 2011. QTL detection for rice grain shape using chromosome single segment substitution lines. Rice Sci. 18(4):273-278.

21. Li, Z., Pinson, S. R. M., Marchetti, M. A., Stansel, J. W., and Park, W. D. 1995. Characterization of quantitative trait loci (QTLs) in cultivated rice contributing field resistance to sheath blight (Rhizoctonia soloni). Theor. Appl. Genet. 91:382-388.

22. Liu, G., Jia, Y., Correa-Victoria, F. J., Prado, G. A., Yeater, K. M., McClung, A., and Correll, J. C. 2009. Mapping quantitative trait loci responsible for resistance to sheath blight in rice. Phytopathology 99:1078-1084. 
23. Liu, G. F., Zeng, R. Z., Zhu, H. T., Zhang, Z. M., Ding, X. H., Zhao, F. M., Li, W., and Zhang, G. Q. 2009. Dynamic expression of nine QTLs for tiller number detected with single segment substitution lines in rice. Theor. Appl. Genet. 118:443-453.

24. Liu, Y. F., Chen, Z. Y., Ji, J. A., and Liu, Y. Z. 2006. Analysis on sheath blight resistance of rice cultivars and candidate cultivars in evaluation test in Jiangsu province. Jiangsu Agric. Sci. 1:27-28.

25. Marchetti, M. A., and Bollich, C. N. 1991. Quantification of the relationship between sheath blight severity and yield loss in rice. Plant Dis. 75:773-775.

26. Marzougui, S., Sugimoto, K., Yamanouchi, U., Shimono, M., Hoshino, T., Hori, K., Kobayashi, M., Ishiyama, K., and Yano, M. 2012. Mapping and characterization of seed dormancy QTLs using chromosome segment substitution lines in rice. Theor. Appl. Genet. 124:893-902.

27. Nelson, J. C., Oard, J. H., Groth, D., Utomo, H. S., Jia, Y., Liu, G., Moldenhauer, K. A. K., Correa-Victoria, F. J., Fjellstrom, R. G., Scheffler, B., and Prado, G. A. 2012. Sheath-blight resistance QTLS in japonica rice germplasm. Euphytica 184:23-34.

28. Pan, X. B., Zhang, Y. F., Zuo, S. M., and Chen, Z. X. 2005. Discussion on QTLs identification and application of important quantitative traits in crops. J. Yangzhou Univ. (Agric. Life Sci. Ed.) 2:50-55.

29. Perchepied, L., Dogimont, C., and Pitrat, M. 2005. Strain-specific and recessive QTLs involved in the control of partial resistance to Fusarium oxysporum $f$. $s p$. melonis race 1.2 in a recombinant inbred line population of melon. Theor. Appl. Genet. 111:65-74.

30. Pinson, R. M. S., Capdevielle, M. F., and Oard, H. J. 2005. Confirming QTLs and finding additional Loci conditioning sheath blight in rice using recombinant inbred lines. Crop Sci. 45:503-510.

31. Shan, J. X., Zhu, M. Z., Shi, M., Gao, J. P., and Lin, H. X. 2009. Fine mapping and candidate gene analysis of spd6, responsible for small panicle and dwarfness in wild rice (Oryza rufipogon Griff.). Theor. Appl. Genet. 119:827-836

32. Sharma, A., McClung, A. M., Pinson, S. R. M., Kepiro, J. L., Shank, A. R., Tabien, R. E., and Fjellstrom, R. 2009. Genetic mapping of sheath blight resistance QTLs within tropical japonica rice cultivars. Crop Sci. 49:256-264.

33. Tan, C. X., Ji, X. M., Yang, Y., Pan, X. Y., Zuo, S. M., Zhang, Y. F., Zou, J. H., Chen, Z. X., Zhu, L. H., and Pan, X. B. 2005. Identification and markerassisted selection of two major quantitative genes controlling rice sheath blight resistance in backcross generations. Acta Genet. Sin. 32(4):399-405.

34. Wang, S., Basten, C. J., and Zeng, Z. B. 2010. Window QTL Cartographer 2.5. Department of Statistical. North Carolina State University, Raleigh, NC. Retrieved 14 September 2011 from http://www.statgen.ncsu.edu/ qtlcart/WQTLCart.htm

35. Wang, S. K., Wu, K., Yuan, Q. B., Liu, X. Y., Liu, Z. B., Lin, X. Y., Zeng, R. Z., Zhu, H. T., Dong, G. J., Qian, Q., Zhang, G. Q., and Fu, X. D. 2012. Control of grain size, shape and quality by OSSPL16 in rice. Nat. Genet. 44(8):950-954.

36. Wang, Y., Pinson, S. R. M., Fjellstrom, R. G., and Tabien, R. E. 2012. Phenotypic gain from introgression of two QTL, qSB9-2 and qSB12-1, for rice sheath blight resistance. Mol Breed. 30:293-303.

37. Wang, Z. B., Zuo, S. M., Li, G., Chen, X. J., Chen, Z. X., Zhang, Y. F., and
Pan, X. B. 2009. Rapid identification technology of resistance to rice sheath blight in seedling stage. Acta Phytopathol. Sin. 2:174-182.

38. Wu, X. J., Zuo, S. M., Chen, Z. X., Zhang, Y. F., Zhu, J. K., Ma, N., Tang, J. Y., Chu, C. C., and Pan, X. B. 2011. Fine mapping of $\mathrm{qSTV} 11^{\mathrm{TQ}}$, a major gene conferring resistance to rice stripe disease. Theor. Appl. Genet. 122:915-923.

39. Yamato, T., Kuboky, Y., and Lin, S. Y. 1998. Fine mapping of quantitative trait loci $\mathrm{Hd} 1, \mathrm{Hd} 2$ and $\mathrm{Hd} 3$, controlling heading date of rice, as single Mendelian factors. Theor. Appl. Genet. 97:37-44

40. Ye, G. Y., Liang, S. H., and Wan, J. M. 2010. QTL mapping of protein content in rice using single chromosome segment substitution lines. Theor. Appl. Genet. 121:741-750.

41. Yin, Y. J., Zuo, S. M., Wang, H., Chen, Z. X., Gu, S. L., Zhang, Y. F., and Pan, X. B. 2009. Evaluation of the effect of qSB-9(Tq) involved in quantitative resistance to rice sheath blight using near-isogenic lines. Can. J. Plant Sci. 4:731-737.

42. Young, N. D. 1996. QTL mapping and quantitative disease resistance in plants. Annu. Rev. Phytopathol. 34:479-501.

43. Zhang, K. Z., Ming, H. M., and Li, P. 2008. Identification and analysis of core rice parents' resistance to rice sheath blight in south rice production regions of China. Plant Prot. 34(1):45-48

44. Zhao, M., Zhang, Z., Zhang, S., Li, W., Jeffers, D. P., Rong, T., and Pan, G. 2006. Quantitative trait loci for resistance to banded leaf and sheath blight in maize. Crop Sci. 46:1039-1045.

45. Zou, J. H., Pan, X. B., Chen, Z. X., Xu, J. Y., Lu, J. F., Zhai, W. X., and Zhu, L. H. 2000. Mapping quantitative trait loci controlling sheath blight resistance in two rice cultivars (Oryza sativa L.). Theor. Appl. Genet. 101:569-575.

46. Zuo, S. M., Wang, Z. B., Chen, X. J., Gu, F., Zhang, Y. F., Chen, Z. X., and Pan, X. B. 2009. Evaluation of resistance of a novel rice germplasm YSBR1 to sheath blight. Acta Agron. Sin. 4:608-614.

47. Zuo, S. M., Yin, Y. J., Pan, C. H., Chen, Z. X., Zhang, Y. F., Gu, S. L., Zhu, L. H., and Pan, X. B. 2013. Fine mapping of qSB-11 ${ }^{\mathrm{LE}}$, the QTL that confers partial resistance to rice sheath blight. Theor. Appl. Genet. 126:12571272 .

48. Zuo, S. M., Yin, Y. J., Zhang, L., Zhang, Y. F., Chen, Z. X., Gu, S. L., Zhu, L. H., and Pan, X. B. 2011. Effect and breeding potential of $\mathrm{qSB}-11^{\mathrm{LE}}$, a sheath blight resistance quantitative trait loci from a susceptible rice cultivar. Can. J. Plant Sci. 1:191-198.

49. Zuo, S. M., Zhang, L., Wang, H., Yin, Y. J., Zhang, Y. F., Chen, Z. X., Ma, Y. Y., and Pan, X, B. 2008. Prospect of the QTL-qSB-9 ${ }^{\text {Tq }}$ utilized in molecular breeding program of japonica rice against sheath blight. J. Genet. Genomics 35:499-505.

50. Zuo, S. M., Zhang, Y. F., Chen, Z. X., Chen, X. J., and Pan, X. B. 2010 Current progress on genetics and breeding of rice resistance to sheath blight. Sci. Sin. Vitae 11:1014-1023.

51. Zuo, S. M., Zhang, Y. F., Yin, Y. J., Chen, Z. X., and Pan, X. B. 2006. Establishment and improvement of inoculation technique and rating system in researching rice sheath blight resistance in field. J. Yangzhou Univ. (Agric. Life Sci, Ed.) 4:57-61. 